\title{
Circulating Neuregulin-1 $\beta$ Level in Psoriatic Patients and its Relation to the Cardiac Function
}

\author{
A.A.Ebrahim, A.A.Ali, A.O.Samir,A.S.IbrahimandA.A.Kamal \\ Dermatology, Venereology and Andrology Dept., Faculty of Medicine, Benha Univ., Benha, Egypt \\ E-Mail:drayaayman3@gmail
}

\begin{abstract}
Psoriasis vulgaris is a chronic inflammatory skin condition, associated with both a physical and a psychological burden .psoriasis is portrayed by erythematous plaques covered with slivery scales over the extensor surfaces, scalp, and lumbosacral locale . Neuregulin-1 $\beta$ (NRG-1) is a layer bound or discharged development and separation factor that intercedes its activity by official to ErbBreceptors. The point of our work is to evaluate serum level of neuregulin $1-\beta$ in psoriatic patients, trying to clarify its part in the sickness pathogenesis and its connection with the illness seriousness and its connection to cardiovascular capacity, foundational and pneumonic hypertension in psoriatic patients. Case control study included 40 patients with psoriasis (Group A). What's more 40 clearly sound controls of coordinated age and sex (Group B). Finding of psoriasis was made based on the patient's set of experiences and the ordinary clinical highlights. Serum NRG-1 levels estimated by chemical connected immunosorbent test (ELISA) . We found that complete psoriasis bunch demonstrated essentially higher serum NRG-1 when contrasted with control gathering, and NRG-1 expanded bit by bit with expanded seriousness of psoriasis . No critical contrasts were found in serum NRG-1 fixation as per sex, smoking and hypertension . NRG-1 indicated huge positive relationship with PASI and seriousness; huge positive connection with pneumonic course systolic weight (PASP) ; critical negative relationship with launch division $(\mathrm{EF})$.
\end{abstract}

\section{Introduction}

Psoriasis is a persistent provocative skin condition, It has been seen as a condition that shows exclusively in the skin. Notwithstanding, the foundational fiery nature of this infection has been affirmed by the presence of a wide exhibit of dysregulated cytokines and incendiary markers in the serum of these patients [1]. Psoriasis is multifactorial sickness with complex hereditary inclination, The pathogenesis of psoriasis is by all accounts driven by the association between natural resistant cells, versatile invulnerable cells and keratinocytes, in a cycle intervened by cytokines (counting interleukins (IL)- 17 and IL-22, tumor corruption factor and other flagging atoms). This prompts a provocative cycle with expanded expansion of epidermal cells, neo-angiogenesis and penetration of dendritic cells in the skin [2].

In psoriatic skin, the change from basal KCs to corneocytes happens in around 5 days, in correlation with the approximately 50-day measure in solid skin [3] . Epidermal hyperplasia, a sign of psoriatic plaques, is related with STAT3 actuation and in a roundabout way managed by IL-17 through the acceptance of IL19 as well as IL-36 by keratinocytes [4].

Countless examinations uncovered an expanded degree of proangiogenic mixes inside psoriatic skin including hypoxia-inducible factor 1 (HIF-1), TNF- $\alpha$, endothelial cell animating angiogenesis factor (ESAF), platelet-determined development factor (PDGF), IL-8, IL-17, angiopoietins and VEGF delivered chiefly by actuated basal keratinocytes [5]

Psoriasis, a constant provocative skin problem, is related with uplifted cardiovascular danger, expanded vascular aggravation (VI), and expanded occurrence of early cardiovascular occasions including myocardial localized necrosis and stroke [6]. Psoriasis being a fundamental provocative state may subsequently help comprehend the function of persistent irritation in atherosclerosis in vivo.

Neuregulin-1 (NRG-1) has a place with the group of epidermal development factor-like proteins, encoded by 4 neuregulin qualities. It is principally dispersed in the anxious and cardiovascular frameworks. NRG-1 flagging is transduced by the ErbB family, which has a place with the receptor protein tyrosine kinase family, and phosphorylation and dimerization of these receptors prompts actuation of downstream pathways [7].

NRG is delivered by cells of endothelial, mesenchymal, and neuronal beginning, while ErbB receptors are found near the ligand, producing neighborhood autocrine, paracrine, or even juxtacrine actions [8]. NRG-1 follows up on different targets, for example, heart, veins, nerves and interstitial liquid, and secures the myocardium, advancing the improvement of cardiovascular capacity through a joined multifaceted impact. It might have potential and significant clinical centrality for the treatment and forecast of sepsis [7].

NRG-1 initiated a quick and transient expansion in tyrosine phosphorylation of TYK2 and JAK3, however not JAK1 or JAK2, and incited STAT3 and STAT5 tyrosine phosphorylation. Upon phosphorylation, STAT3 moved to the core inside $1 \mathrm{~h}$. Actuation of the JAK-STAT pathway was reliant on HER2/HER3heterodimerization and was essential for NRG-1-instigated multiplication [9].

Dynamic STAT3 is commonly present both in psoriatic resistant cells and in psoriatic keratinocytes prompting epidermal hyperplasia [10].

The point of our work is to survey serum level of neuregulin $1-\beta$ in psoriatic patients, trying to explain its part in the illness pathogenesis and its connection with the infection seriousness and its connection to 
cardiovascular capacity, foundational and aspiratory hypertension in psoriatic patients.

\section{Patients and methods}

This study was completed on 40 psoriasis patients (Group A) and 40 age and sex coordinated obviously sound people as controls (Group B). All patients recurrited from the outpatient center of Dermatology and Andrology Department of Banha University Hospitals in the period between January 2019 to January 2020. Educated assents were taken from all subjects remembered for the study.The study was endorsed by the nearby morals advisory group on research including human subjects of Benha workforce of medication.

Itemized dermatological assessment to assess clinical variation, seriousness of psoriasis and reject the presence of other skin sicknesses.

Determination of psoriasis was made based on the patient's set of experiences and the common clinical highlights of psoriasis vulgaris which shows too differentiated erythematous plaques covered by gleaming white scales, ordinarily circulated evenly on the scalp, elbows, knees, and lumbosacral territory.

The absolute seriousness score was gotten from PASI score, the body was separated into four areas and each segment had a coefficient relying upon its expansion (to be specific, 0.1 head, 0.2 upper appendages, 0.3 trunks, 0.4 lower appendages). Every zone was scored independently, considering the qualities of the plaque (erythema, induration/thickness and scaling) and, at that point, the scores were amounted to give a the last score.

On this premise, psoriasis seriousness was characterized utilizing the accompanying standards: gentle, PASI $<7$ and DLQI $<7$; moderate, PASI=7-15 and DLQI=5-15 (delegated extreme when hard to-treat destinations are influenced or when there is a huge psychosocial sway); serious, PASI $>15$, autonomously of the DLQI score.

This pack utilizes compound connected insusceptible sorbent examine (ELISA) in view of the Biotin twofold counter acting agent sandwich innovation to test the Human Neuregulin 1 (NRG-1). Add Neuregulin 1(NRG-1)to the wells, which are precovered with Neuregulin 1(NRG-1)monoclonal immunizer and afterward hatch. From that point onward, add hostile to NRG-1 antibodies marked with biotin to join with streptavidin-HRP, which structures insusceptible complex. Eliminate unbound catalysts after brooding and washing. Add substrate An and B. At that point the arrangement will transform blue and change into yellow with the impact of corrosive. The shades of arrangement and the centralization of Human Neuregulin 1 (NRG-1) are emphatically related. Test well to be tried: Add $40 \mu$ l example and afterward $10 \mu 1$ NRG-1 antibodies, 50 $\mu$ streptavidin-HRP. At that point cover it with seal plate film. Shake delicately to blend them up. Brood at $37^{\circ} \mathrm{Cfor} 60$ minutes.Preparation of washing arrangement: Dilute the washing focus (30X) with refined water for sometime in the future. Washing by eliminate the seal plate layer cautiously, channel the fluid and shake off the excess fluid. Fill each well with washing arrangement. Channel the fluid following 30 seconds' standing. At that point rehash this method multiple times and blotch the plate.

Shading improvement: Add 50 $\mu$ l chromogen arrangement An initially to each well and afterward add $50 \mu \mathrm{l}$ chromogen arrangement B to each well also. Shake tenderly to blend them up. Hatch for 10 minutes at $37^{\circ} \mathrm{C}$ away from light for shading advancement.

Add $50 \mu$ l Stop Solution to each well to stop the response (the blue shading changes into yellow quickly at that point).

Accept clear well as zero, measure the absorbance (OD) of each well individually under 450nm frequency, which ought to be done inside the 10 minutes subsequent to having added the stop arrangement.

As per guidelines' focuses and the comparing OD esteems, ascertain the direct relapse condition of the standard bend. At that point as per the OD estimation of tests, ascertain the convergence of the comparing test. Extraordinary programming could be utilized to ascertain too.

The gathered information was changed, coded and classified utilizing factual bundle for Social Science (IBM Corp. Delivered 2017. IBM SPSS Statistics for Windows, Version 25.0. Armonk, NY: IBM Corp.). Information were introduced and reasonable investigation was finished by the kind of information got for each parameter.

\section{Results}

Table (1) Comparison ofvariables between all studied groups.

\begin{tabular}{|c|c|c|c|c|c|c|}
\hline Variable & & & $\begin{array}{l}\text { ntrol } \\
=40 \\
n \pm \text { SD }\end{array}$ & $\begin{array}{r}\text { Pso } \\
\mathrm{Me}\end{array}$ & $\begin{array}{l}\text { asis } \\
\text { SD } \\
\text { SD }\end{array}$ & $\mathrm{P}$ \\
\hline Lipid profile & $\begin{array}{l}\text { Triglycerides }(\mathrm{mg} / \mathrm{dL}) \\
\text { Cholesterol }(\mathrm{mg} / \mathrm{dL})\end{array}$ & $\begin{array}{c}90 \\
125.6\end{array}$ & $\begin{array}{c}18 \\
24.4\end{array}$ & $\begin{array}{c}92.2 \\
156.9\end{array}$ & $\begin{array}{l}31.2 \\
37.3\end{array}$ & $\begin{array}{l}0.309 \\
<0.001\end{array}$ \\
\hline $\begin{array}{l}\text { Systemic blood pressure } \\
\text { Echocardiographic } \\
\text { parameters }\end{array}$ & $\begin{array}{c}\text { Ejection fraction }(\mathrm{EF})(\%) \\
\text { Pulmonary artery systolic } \\
\text { pressure (PASP) }\end{array}$ & $\begin{array}{c}7 \\
68.4\end{array}$ & $\begin{array}{c}17.5 \% \\
4.2 \\
26.7 \pm 8.3\end{array}$ & $\begin{array}{c}11 \\
62.8\end{array}$ & $\begin{array}{c}27.5 \% \\
4.7\end{array}$ & $\begin{array}{l}0.284 \\
0.614 \\
0.040\end{array}$ \\
\hline
\end{tabular}


SD, standard deviation; lipid profile are is compared using t test,EF and PASP is compared using $\mathrm{t}$ test; systemic blood pressure is compared using chi square test.

Lipid profile, Serum cholesterol level was significantly higher in psoriasis patients than control group $(\mathrm{P}<0.001)$.No significant differences were found in serum triglyceride $\mathrm{TG}$ level and systemic blood pressure between both studied groups $(\mathrm{P}>0.05)$.Ejection fraction $(\mathrm{EF})$ was non-significantly lower in psoriasis patients than control group. Pulmonary artery systolic pressure (PASP) was significantly higher in psoriasis group when compared to control group $(\mathrm{P}$ value $=0.040)$.

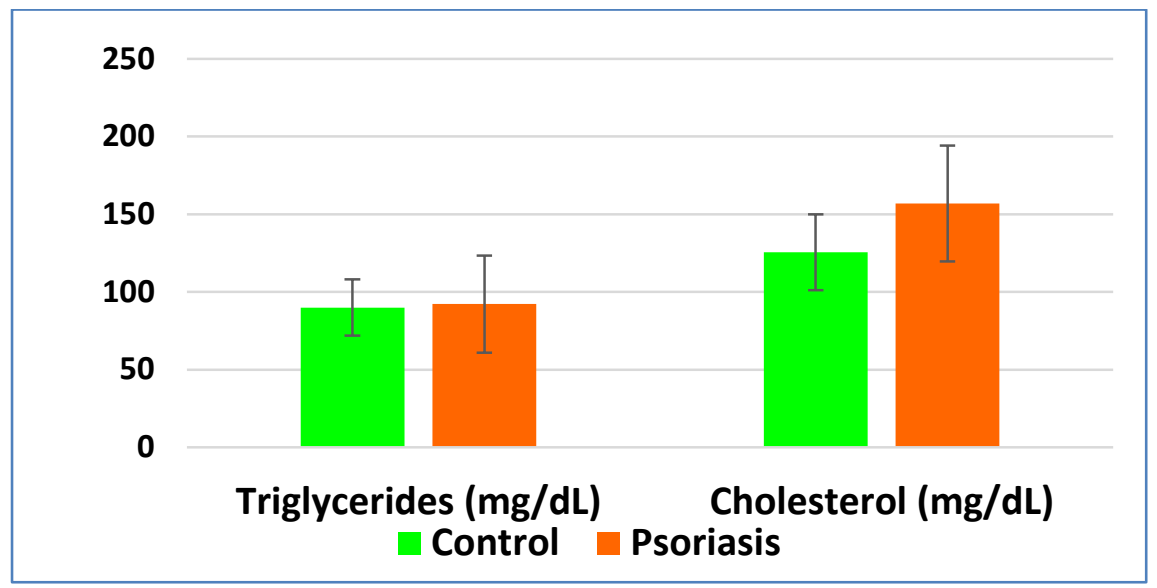

Fig (1) Lipid profile between both studied group of patients.

\section{Discussion}

Psoriasis is achronic inflammatory disease that is commonly encountered in primary care and is associated with significant morbidity that extends beyond the skin manifestations [11].It affects $1 \%$ to $3 \%$ of the population worldwide [12].

Much effort has been made in defining the aetiopathogenesis of the inflammatory/autoimmune systemic diseases of the skin as psoriasis. It is widely accepted that these are strictly associated to an imbalance between regulatory (Treg) and effector $\mathrm{T}$ (Th1/Th2 and Th17) responses. IL-10 producing T regs play a crucial role in restoring and maintaining the physiologic and delicate Th1/Th2 and Th17 balance[13].The T-cell infiltrate and dendritic cell subpopulations present in lesional areas mainly express cytokines IFN- $\gamma$, TNF- $\alpha$, IL-17 and IL-22, which induce keratinocytes to further overexpress inflammatory mediators. This causes the chronic skin inflammation that is responsible for the proliferation of keratinocytes and endothelial cells, neo-angiogenesis and infiltration of dendritic cells[14].

Neuregulin-1 $\beta$ (NRG-1) is a membrane-bound or secreted growth and differentiation factor that mediates its action by binding to ErbB receptors [15].

NRG-1 acts on multiple targets such as heart, blood vessels, nerves and interstitial fluid[16].It is released by cells of endothelial, mesenchymaland neuronal origin[17].

NRG regulate the cardiac myocyte responses, including cell survival, growth, adaptation to stress and proliferation, emerging data support a broader role for NRGs in the regulation of metabolism, inflammation, and fibrosis in response to injury[18].
The role of NRG1 in psoriasis is not fully known. Therefore, the aim of our study was to evaluate the serum levels of NRG1 in patients with psoriasis in comparison to age and sex matched healthy controls and their correlation with the severity of psoriasis in a trial to provide an idea about its possible role in the pathogenesis of psoriasis and its relation to cardiac function in those patients.

\section{Conclusion}

From the aftereffect of present investigation, it is directed that serum NRG-1 Beta may assume apositive part in psoriasis pathogenesis . in addition , it very well may be viewed as a biomarker for psoriasis and its seriousness with high affectability, explicitness and precision.Also it might play role in cardiac diseases.

\section{References}

[1] M.J.E.Visser , D.B.Kell, E. Pretorius"Bacterial Dysbiosis and Translocation in Psoriasis Vulgaris." Frontiers in cellular and infection microbiology, Vol. 9 (7), PP.4, 2019.

[2] A.M. Martins, A.Ascenso, H.M.RibeiroThe BrainSkin Connection and the Pathogenesis of Psoriasis, A Review with a Focus on the Serotonergic System. Cells, Vol.9(4), PP.796,2020.

[3] F.Benhadou, D.Mintoff, V. del MarmolPsoriasis: Keratinocytes or immune cells-Which is the trigger?,Dermatology; vol. 23(5), pp. 91100,2020 .

[4] J .Kim ,J.G. Krueger Highly Effective New Treatments for Psoriasis Target the IL-23/Typ17 T Cell Autoimmune Axis , Annu Rev Med,Vol.68, PP.255-69, 2017.

[5] G.G. Teixeira, N.L. Mari, J.C.C.de Paula 
Cell adhesion molecules, plasminogen activator inhibitor type 1 , and metabolic syndrome in patients with psoriasis.ClinExp Med;Vol.20, PP.39-48.2020.

[6] H.B. Naik, B .Natarajan, E .Stansky

Severity of psoriasis associates with aortic vascular inflammation detected by FDG PET/CT and neutrophil activation in a prospective observational study.ArteriosclerThrombVascBiol;Vol.35(12) , PP.2667-2676,2015.

[7] S.Salvany, A. Casanovas, O .TarabalLocalization and dynamic changes of neuregulin-1 at C-type synaptic boutons in association with motor neuron injury and repair. FASEB J, Vol.33 , PP.78337851,2019

[8] A .Gumà, V.Martínez-Redondo, I.López-Soldado Emerging role of neuregulin as a modulator of muscle metabolism. Am J PhysiolEndocrinolMetab, Vol.298(4) , PP.742750.2010 .

[9] J.Liu , J.A. Kern Neuregulin-1 activates the JAKSTAT pathway and regulates lung epithelia cell proliferation. Am J Respir Cell MolBiol, Vol. 27(3) , PP.306-13,2002.

[10] A. K.Alves de Medeiros ,R.Speeckaert, E.DesmetJAK3 as an emerging target for topical treatment of inflammatory skin diseases. PLoSOne , Vol.11, pp. 164-80,2016.

[11]Tom Kovitwanichkanont, Alvin Chong , Peter Foley Skin health institute ,Melbourne ,VIC Med J Aust, Vol. 212(11), PP.528-534,2020.
[12] L .Eder, J.Widdifield, C.F. Rosen Trends in the Prevalence and Incidence of Psoriasis and Psoriatic Arthritis in Ontario, Canada: A Population-Based Study.Arthritis Care Res (Hoboken), Vol.71(8), PP.1084-1091,2019

[13] C.Lanna, M. Mancini, R.GazianoSkin immunity and its dysregulation in psoriasis. Cell Cycle, Vol.18(20), PP.2581-2589,2019.

[14]P.Gisondi, M.Del Giglio,G.GirolomoniTreatment approaches to moderate to severe psoriasis. Int. J. Mol. Sci, Vol.18, PP.24-27,2017.

[15]E.Boateng, J.T.deKay, S.M.Peterson High ErbB3 activating activity in human blood is not due to circulating neuregulin-1 beta.Life Sci, Vol.251 ,pp117-634,2020.

[16]S.Salvany, A.Casanovas, O.TarabalLocalization and dynamic changes of neuregulin-1 at C-type synaptic boutons in association with motor neuron injury and repair. FASEB journal : official publication of the Federation of American Societies for Experimental Biology , Vol.33, PP.7833-7851,2019.

[17] A.Gumà, F.Díaz-Sáez, M.Camps Neuregulin, an Effector on Mitochondria Metabolism That Preserves Insulin Sensitivity.Frontiers Physiol, Vol.11, PP.696,2020.

[18] A.Geissler , S.Ryzhov, D. B. Sawyer Neuregulins: protective and reparative growth factors in multiple forms of cardiovascular disease. Clinical science (London, England : 1979), Vol.134(19), PP.2623-2643,2020. 